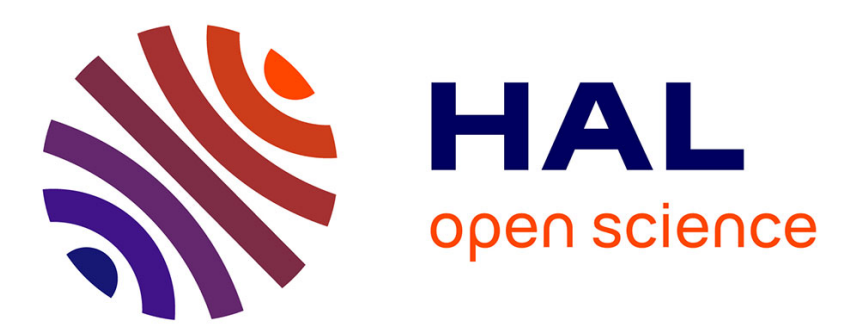

\title{
Multiscale Techniques for the Detection of Precipitation Using Thermal IR Satellite Images
}

\author{
Antonio Turiel, Jacopo Grazzini, Hussein Yahia
}

\section{To cite this version:}

Antonio Turiel, Jacopo Grazzini, Hussein Yahia. Multiscale Techniques for the Detection of Precipitation Using Thermal IR Satellite Images. IEEE Geoscience and Remote Sensing Letters, 2005, 2 (4), pp.447-450. 10.1109/LGRS.2005.852712 . inria-00423740v2

\section{HAL Id: inria-00423740 \\ https://hal.inria.fr/inria-00423740v2}

Submitted on 18 Feb 2014

HAL is a multi-disciplinary open access archive for the deposit and dissemination of scientific research documents, whether they are published or not. The documents may come from teaching and research institutions in France or abroad, or from public or private research centers.
L'archive ouverte pluridisciplinaire HAL, est destinée au dépôt et à la diffusion de documents scientifiques de niveau recherche, publiés ou non, émanant des établissements d'enseignement et de recherche français ou étrangers, des laboratoires publics ou privés. 


\title{
AUTHOR VERSION
}

\section{Multiscale Techniques for the Detection of Precipitation Using Thermal IR Satellite Images}

\author{
Antonio Turiel, Jacopo Grazzini, and Hussein Yahia
}

\begin{abstract}
It is thought that satellite thermal infrared (IR) images can aid to the detection of precipitation, an interesting possibility due to the existence of geostationary satellites with thermal IR sensors which would enable a good spatial and temporal tracking of rain and storms. In this letter, we explore the application of multiscale/multifractal techniques in the design of new methods for the assessment and tracking of pluviometry. We first identify the main streamlines by a singularity analysis of the wavelet projections of the IR record. From the streamlines, we derive a proxy scalar image that represents the result of pure horizontal advection. From the comparison of original and proxy we localize the places at which horizontal advection fails, which we identify with convection places. We illustrate our methodology with thermal IR images from Metosat acquired during heavy tropical rainfall, and compare the results with some data from the Tropical Rainfall Measuring Mission satellite.
\end{abstract}

Index Terms-Edge detection, fractal, multifractal, multiscale processing, rain detection.

\section{INTRODUCTION}

W ITH the advent today of the most sophisticated networks of sensors and reliable modeling software, the problem of rainfall detection from earth observation data is becoming crucial and challenging. A solid knowledge of the region under study, of its seasonal variability, and of its meteorology will aid to cast good overal predictions from scarce data, but precipitation is much more elusive to forecast, due to its intermittent character both in space and time.

A reasonable alternative to ground stations when these are not available, and in any instance as an interesting source of complementary data, is satellite imagery [1]-[5]. Satellites provide synoptic views of large areas and transmit data at a steady rate. The main problems with satellite images in the context of meteorology come from that they only give access to upper layers of phenomena taking place in three dimensions

Manuscript received January 11, 2005; revised April 27, 2005. The work of A. Turiel was supported by a Ramón y Cajal contract from the Spanish MoE. $\mathrm{J}$. Grazzini is now supported by a Marie-Curie postdoctoral fellowship and formerly by a grant from the Regional Council of Île-de-France (E.1358).

This letter has supplementary downloadable material available at http://ieeexplore.ieee.org, provided by the authors. This includes six animated GIF files, containing the original sequences that have been processed in the ellaboration of this work. This material is $12.4 \mathrm{MB}$ in size.

A. Turiel is with the Institut de Ciències del Mar, 08003 Barcelona. Spain (e-mail: turiel@icm.csic.es).

J. Grazzini is with the Regional Analysis Division, Institute of Applied and Computational Mathematics, Foundation for Research and Technology, 71110 Heraklion, Crete, Greece (e-mail: grazzini@iacm.forth.gr).

H. Yahia is with the Institut National de Recherche en Informatique et an Automatique Rocquencourt, 78153 Le Chesnay Cedex, France (e-mail: Hussein.Yahia@inria.fr).

Digital Object Identifier 10.1109/LGRS.2005.852712 and that the observed variables are not always easy to link with the thermodynamics of atmosphere. However, the atmosphere is a strongly stratified flow: gravity and Earth's rotation play a role in the stratification process, but it is the thermodynamics and in particular radiation (received or reemitted) of the atmosphere (and the surface) which mostly determine temperature profiles, stability, and, in-fine, stratification. Hence, for many regions and thermodynamical conditions, mainly at the time and spatial scales of the quasi-geostrophic approximation, atmosphere motion can be approximated by that of a horizontal two-dimensional (2-D) fluid. But rain is precisely an essentially three-dimensional (3-D) phenomenon, so evidences of precipitation can only be indirectly inferred from satellite images.

Studies conducted in the very instable intertropical convergence zone (ITCZ) have shown that in the case of convective structures, cloud top temperature is a key parameter linked to precipitation [6], hence motivating researchers on the determination of threshold values in satellite infrared (IR) images for rainfall determination [2], [4], [6], [7]. These studies justify the focus taken in this letter: we will determine convection in large cumulo-nimbus systems in the ITCZ. Cumulo-nimbus form the so-called convective towers, which are strong jets of air moving vertically, making a boiling effect on top of clouds. Within the ITCZ, the detection of a convective tower is almost equivalent to the detection of a rainfall event. Ice creation on top the convective towers is intense. Then, satellites operating in a hyperfrequency range interacting with ice-crystal molecular bounds will be able to detect ice, hence the convective towers and so precipitation. The Tropical Rainfall Measuring Mission (TRMM) provides hyperfrequency images in several useful ranges to detect water and ice [8], [9]. But it has important drawbacks: first, its swath is quite narrow, and second, as it operates with high-frequency radiation, it cannot be as far from ground as geostationary satellites. Consequently, its temporal acquisition rate is too low when compared to the duration of rainfall events.

Geostationary satellites provide continuous-time acquisition and wide swaths, but yield detectors which operate at shorter wavelengths, from IR to visible [2], [10]. Visible images provide no information during the night, so IR images are possible candidates to produce a steady track of precipitation [7], [11]. We will concentrate on thermal IR images (namely, images acquired in the thermal IR channel, with wavelength 10.5-12.5 $\mu \mathrm{m}$, onboard the Meteosat satellite) because one of the signs of convection is heat exchange, which should induce thermal gradients that we will try to detect. In addition, temperature behaves as a diffusive (rather passive) scalar, so it can be locally employed to track motion, provided the effect of diffusion has been taken into account.

\section{AUTHOR VERSION}




\section{AUTHOR VERSION}

The work presented herein is an image and a complex signal model for the analysis of turbulent data, applied to the meteorological context specified above. This letter presents the model part, along with simple verification of its results with some TRMM data: the quantitative validation will be the subject of another study.

\section{Assessment of the MaIn CURREnt Lines}

The first step to characterize convection areas is to identify streamlines. As we have only access to a 2-D planar projection, the streamlines that we could eventually detect are plane projections of the actual 3-D streamlines (a fact that will be useful later to characterize convection). In order to extract them, we will make use of wavelet techniques for singularity characterization [12]. Let the IR intensity at any point $\vec{x}$ in the image plane to be the 2-D scalar function $s(\vec{x})$; we compute its gradient $\nabla s(\vec{x})$ and classify the points according to the local singularity strength of this quantity. More precisely, we study the behavior of the wavelet projections $T_{\Psi}|\nabla s|(\vec{x}, r)$, defined as

$$
T_{\Psi}|\nabla s|(\vec{x}, r) \equiv \int d \vec{x}^{\prime}|\nabla s|\left(\vec{x}^{\prime}\right) \frac{1}{r^{2}} \Psi\left(\frac{\vec{x}-\vec{x}^{\prime}}{r}\right) .
$$

Here $\Psi$ is the analysis wavelet, which can be chosen in a more or less wide family, depending on the requirements on the signal [13]. The point $\vec{x}$ appearing in (1) designs the pixel under analysis, while $r$ is a scale parameter which allows to zoom in and out around $\vec{x}$ and so to study the relation of the gradient of $s$ at $\vec{x}$ with those of surrounding points. The signal $s(\vec{x})$ will be multifractal if at any point $\vec{x}$ all the wavelet projections centered on $\vec{x}$ depend on $r$ as a power-law, i.e.,

$$
T_{\Psi}|\nabla s|(\vec{x}, r) \approx \alpha(\vec{x}) r^{h(\vec{x})}(r \rightarrow 0) .
$$

The exponent $h(\vec{x})$ is called singularity exponent and characterizes the local degree of regularity of the signal at that point [12]-[14]. Due to thermodynamical reasons, turbulent flows are of multifractal character [15], [16] and, thus, we expect any meaningful intensive variable defined on such flows to exhibit a multifractal behavior [17].

Singularities allow to classify points, as points with the same singularity exponent obey the same scale transformation rules and are characteristics of the flow. The decomposition of the image in the different singularity components is known as multifractal decomposition [12], as these sets are fractal with different fractal dimensions (this is the reason for the name multifractal). The interest of multifractal decomposition comes from the fact that it is rather well conserved by flow advection [18].

Among all the fractal components, one of them seems the most interesting: the so-called most singular manifold (MSM). This set comprises the points with strongest singularity (i.e., most negative value), and so it concentrates on the fronts of the signal (in the sense of transition fronts, not to be confused with atmospheric fronts although in some cases they may coincide). The existence of a most negative value must be addressed: we note that the singular exponents $h(\vec{x})$ are related to singularities discussed in recent studies which make use of multiplicative cascade models. These models use an assumption of stationarity; consequently the integral over a scale-dependent spatial domain of the temperature gradient is strongly related to the average rate of kinetic energy dissipation per unit mass, which displays a multifractal behavior [15]. Multiplicative cascade theories for the modeling of rain precipitation have firmly gained ground in recent years, and they fit in two classes: discrete in scale multiplicative cascades [19] and continous in scale multifractals, from which universal multifractals are the most important subclass [20]. The former makes no assumption on the cascade generator (except of being scale discrete), while the latter uses multiplicative cascades, generated by stable-laws in the case of universal multifractals (e.g., Gaussian or Lévy laws) which can lead to unbounded singularities. The use of universal multifractals generated by extremal Lévy laws or even infinitely divisible process as Log-Poisson multiplicative cascades (see [20]) lead to a lower bound on the order of singularities, and they adequately model a wide range of multiscale phenomena. Moreover, experiments described in [21] on Meteosat data have shown lower bounds that cannot be interpreted as an effect of resolution.

Fully developed thermal transitions are in equilibrium with flow advection [16], and thus singularity fronts coincide with some streamlines. Notice that in general the isotransition lines (i.e., the MSM) are not isotherms; isotransition lines follow the advective direction even in cases in which baroclinic instabilities are important [18]. As a striking experimental validation, empirical MSMs always consist of curve-like, one-dimensional separate parts, which according to the discussion above must correspond to streamlines. Hence, isolating the MSM is equivalent to isolate some important instantaneous streamlines of the flow. The validity of this construction relies in three key hypotheses; namely, the turbulence is fully developed [16], the signal is multifractal [21], and the singularity components (and in particular thermal transitions) are conserved [18]. Under these three conditions, we have access to an instantaneous determination of streamlines, without having to process a sequence: this is one of the virtues of our method. In Fig. 1 (right) the obtained MSMs are shown. The MSMs comprise obvious contours of clouds (which are indeed thermal fronts) and some less obvious filaments.

\section{REDUCED IMAGE AND COMPARISON WITH DATA}

Once the MSM streamlines have been detected (using the previously discussed methodology or any other reasonable alternative), we will generate a new signal which represents the effect of pure 2-D advection. To do that, we will reconstruct the image by using a deterministic algorithm which is compatible with the statistical properties of fully developed turbulence. This algorithm was introduced in [22] and was first applied to IR images, with success, in [21]. The starting data for the algorithm are the gradient vectors over the MSM only. In order to generate our "advective signal" (that we will call the reduced image) we will impose some constraints on the starting data to fulfill the requirements of pure advection. If the reduced image is being advected by the flow, its gradients must be orthogonal to the streamlines, so we will impose that the starting data (gradient over the MSM) will be unitary vectors, perpendicular to 


\section{AUTHOR VERSION}
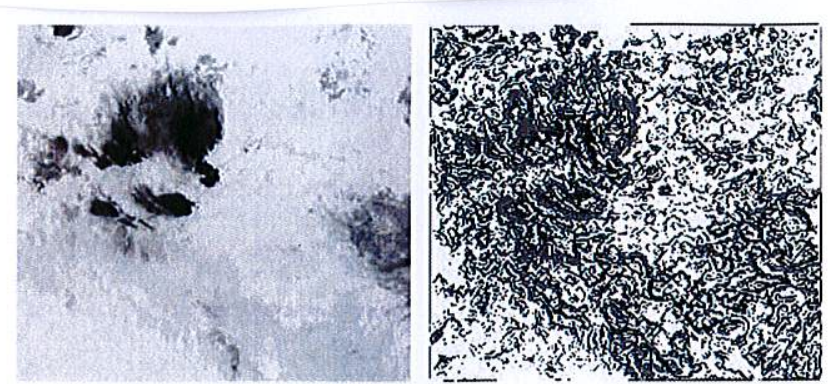

Fig. 1. (Left) Meteosat thermal IR sequence. Area: from $2^{\prime} 15^{\prime \prime}$ to $25^{\prime}$ east, and from $0^{\prime}$ to $20^{\prime}$ north. Images were acquired during July 31, 1998, at a temporal resolution of an hour. Graylevel values are temperatures, in Kelvin degrees. (Right) MSM sequence, taken as the $40 \%$ most singular points in the image. MSM points are marked in black and gray, to keep track of gradien orientation. A pair of supplementary animated GIFs is available for download at http://ieeexplore.ieee.org. The files are $2.7 \mathrm{MB}$.
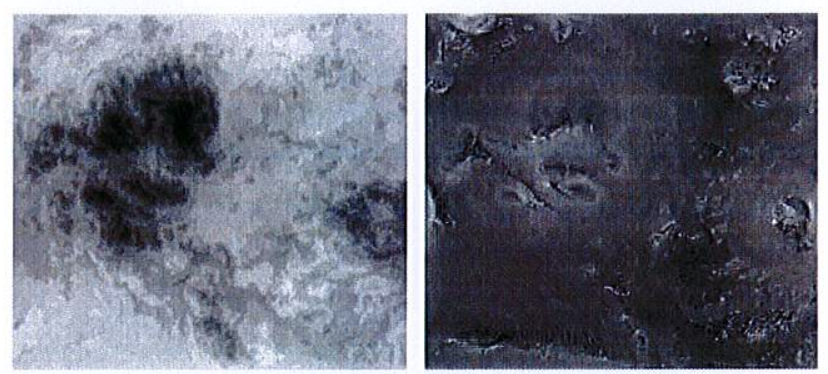

Fig. 2. (Left) Reduced image sequence, obtained from the current lines presented in Fig. 1, right. (Right) Sources image sequence. White: poles, black: zeroes. A pair of supplementary animated GIFs is available for download at http://ieeexplore.ieee.org. The files are $4.7 \mathrm{MB}$.

the MSM, and with the same orientation as the original gradients. We need to keep track of orientation as this is a global feature and cannot be calculated locally. We impose the vectors to be unitary because we lack of any additional information, and this value ensures conservation under flow advection. In Fig. 2 (left), we show the result for the Meteosat sequence (see [21] for further details).

The reduced images have a much more equalized distribution of graylevels and represent how temperature (in the sense of thermal IR radiance) would look if it had been horizontally advected (i.e., transported inside the image plane). For the advection-dominated areas, we expect to observe a proportionality between original data and reduced image. It should be noticed that, as the reduced image has no units, we need a scale factor to relate the values of original and reduced images. For advection-dominated regions, we should expect a relation of the type $s=a R+b$ where $s$ is the original data, $R$ is the reduced image, and $a, b$ are constants. Such a simple expression allows us to classify points according to the degree of advection/convection: we perform local linear regressions of the expression $s(\vec{x})=a R(\vec{x})+b$ over neighborhoods of each point $\vec{x}$. We implement a multiscale version of the linear regression, i.e., each point in the image is used for the regression, but it is given a weight which depends on the distance $d$ to the central point as $d^{-k}$; a value of $k=2$ was used in the present calculation. This way, we can assign to each point the value of the regression coefficient $\rho(\vec{x})$ which measures how well the linear relation holds. The experimental values of $\rho$ are always positive and
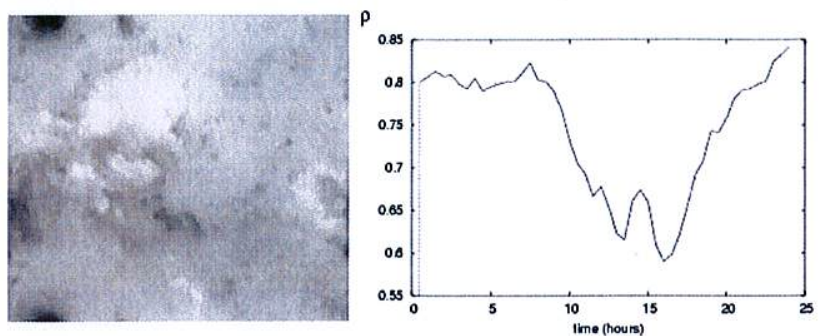

Fig. 3. (Left) Regression coefficient image sequence. The greater correlation, the brighter the point. (Right) Time evolution of the spatial mean of the regression coefficient. A supplementary animated GIF is available for download at http://ieeexplore.ieee.org. The file is $2.4 \mathrm{MB}$.

range from 0-0.999; you can see a graylevel representation of them in Fig. 3. The darkest points correspond to the less correlated areas, which by the discussion above correspond to the convection-dominated areas.

The analysis of the correlation image reveals that the correlation between original image and reduced one drops when and where strong convective systems are being developed. However, due to the need of a local neighborhood for the evaluation of the linear regression, the regression coefficient is anomalously high inside large cloud tops, because they present a narrow dynamic range (as they are very cold) and the contrast is very weak. The weak gradients inside these clouds do not allow to see details inside them with this approach. We need a more localized measure. A natural way to obtain such fine determination is to divide the original image by the reduced signal in order to suppress their common multifractal structure and emphasize their differences. It is the gradient what has been used to characterize singularities equation (2), so we should divide the gradients of the data by those of the reduced image. The linear relation between $s$ and $R$ over advective areas becomes simpler when expressed in terms of gradients: $\nabla s=a \nabla R$. We will obtain the so-called sources term $\sigma(\vec{x})=|\nabla s|(\vec{x}) /|\nabla R|(\vec{x})$, which will give us a better localization of convective movements. As before, we apply a scale-invariant weight to the gradients with $k=1$ in order to improve detection and reduce discretization errors. In Fig. 2, we show the sources terms for the Meteosat sequence. For the advection-dominated regions, the value of the sources term $\sigma$ is constant, while for the zones of strong convection the sources term becomes zero or infinity (pole). As it is observed in the figure, the detection capability is increased with sources, with some new convection focii inside the clouds. In addition, when these images are compared with the scarce TRMM images acquired in the $85.5-\mathrm{GHz}$ channel, we observe a striking correspondence among zeroes/poles and precipitation in many images; see Fig. 4. Since TRMM data are considered as very valuable by meteorologists w.r.t. pluviometry [3], we propose, as a first result, the congruence of source locations with ice crystals locations as given by TRMM data. Quantitative correlation is the subject of future work; see also [23].

\section{CONCLUSION}

In this letter, we have presented a new methodology to localize the places associated to strong convective movements from thermal IR images. In a first stage, the main streamlines 


\section{AUTHOR VERSION}

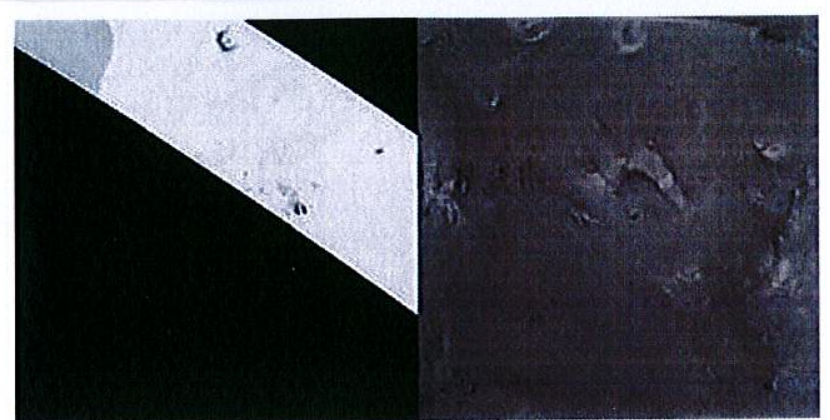

Fig. 4. Comparison between hyperfrequency records from the TRMM satellite (left; black: no acquisition) and sources (right; white: poles, black: zeroes). Pixels in the TRMM images have graylevel values encoding microwave radiation energy. Both sequences were obtained in different, coincident spatial locations and differ at most $15 \mathrm{~min}$. They were acquired during July 31 and August 1, 1998. A supplementary animated GIF is available for download at http://ieeexplore.ieee.org. The file is $2.6 \mathrm{MB}$.

are identified via a classification of points by their singularity strength. The singularity of each point is evaluated using wavelet projections. In the second stage, we generate a reduced image, which corresponds to a scalar variable completely advected by the 2-D flow defined by the streamlines obtained before. Under conditions of advection, there exists a linear relation between the two signals, so we can characterize the existence of strong convection by the deviation from this behavior. The main methodology presented here is based on properties of fully developed turbulence and that it gives an instantaneous determination of convection, without need of processing a time sequence of images. Its main drawbacks are that, in the present stage, this method is rather qualitative, and so far the results have not been validated. As a future work, quantitative correlation with TRMM data will be addressed.

\section{ACKNOWLEDGMENT}

The authors are grateful to the Laboratoire de Météorologie Dynamique (X/Paris) for the data. The authors recognize the support of the MERSEA Project (EU AIP3-CT-2003-502885).

\section{REFERENCES}

[1] V. Levizzani, J. Schmetz, H. Lutz, J. Kerkmann, P. P. Alberoni, and M. Cervino, "Precipitation estimations from geostationary orbit and prospects for Meteosat second generation," Meteorol. Appl., vol. 8, pp. 23-41, 2001.

[2] C. G. Griffith, W. L. Woodley, P. G. Grube, D. W. Martin, J. Stout, and D. N. Sikdar, "Rain estimation from geosynchronous satellite imagery-Visible and infrared studies," Mon. Weather Rev., vol. 106, no. 8, pp. 1153-1171, 1978 .
[3] K. Ramage, "Contribution à l'estimation des précipitations et à la caractérization des nuages en Afrique de l'Ouest à partir des observations des satellites Meteosat et TRMM,' Ph.D. thesis, Univ. Paris VII, Paris, France, 2002.

[4] P. A. Arkin and B. Meisner, "The relationship between largescale convective rainfall and cold cloud over the Western Hemisphere during 1982-1984," Mon. Weather Rev., vol. 115, no. 1, pp. 51-74, 1987.

[5] C. Morel, F. Orain, and S. Senesi, "Automated detection and characterization of MCS using the Meteosat infrared chanel," in Proc. Eumetsat Meteorological Satellite Data Users' Conf., 1997, pp. 213-220.

[6] M. Desbois, T. Kayiranga, and B. Gnamien, "Diurnal cycle of convective cloudiness over tropical Africa observed from Meteosat: Geographic characterization and interannual variations," Ann. Geophys., vol. 7, pp. 395-404, 1989.

[7] R. F. Adler and A. J. Negri, "A satellite infrared technique to estimate tropical convective and stratiform rainfall," J. Appl. Meteorol., vol. 27, no. 1 , pp. 30-51, 1988.

[8] A. J. Negri, R. F. Adler, E. J. Nelkin, and G. J. Huffman, "Regional rainfall climatologies derived from Special Sensor Microwave Imager (SSM/I) data," Bull. Amer. Meteorol. Soc., vol. 75, no. 7, pp. 1165-1182, 1994.

[9] J. Simpson, C. Kummerow, W.-K. Tao, and R. F. Adler, "On the Tropical Rainfall Measuring Mission (TRMM)," Meteorol. Atmos. Phys., vol. 60, pp. 19-36, 1996.

[10] V. Levizzani, "Intense rainfall monitoring from geostationary satellites," in Proc. Conf. Satellites, Meteorology and Oceangraphy, 1998, pp. 327-330.

[11] Y. Arnaud, M. Desbois, and J. Maizi, "Automatic tracking and characterization of African convective systems on Meteosat pictures," J. Appl. Meteorol., vol. 31, pp. 443-493, 1992.

[12] A. Turiel and N. Parga, "The multi-fractal structure of contrast changes in natural images: From sharp edges to textures," Neur: Comput., vol. 12, pp. 763-793, 2000.

[13] A. Arneodo, F. Argoul, E. Bacry, J. Elezgaray, and J. F. Muzy, Ondelettes, Multifractales et Turbulence. Paris, France: Diderot Editeur, 1995.

[14] Z. R. Struzik, "Determining local singularity strengths and their spectra with the wavelet transform," Fractals, vol. 8, no. 2, pp. 163-179, Jun. 2000 .

[15] G. Parisi and U. Frisch, "On the singularity structure of fully developed turbulence," in Turbulence and Predictability in Geophysical Fluid Dynamics. Proc. Int. School of Physics E. Fermi, M. Ghil, R. Benzi, and G. Parisi, Eds., Amsterdam, North Holland, 1985, pp. 84-87.

[16] U. Frisch, Turbulence. The Legacy of Kolmogorov. Cambridge, U.K.: Cambridge Univ. Press, 1995.

[17] J. Arrault, A. Arneodo, A. Davis, and A. Marshak, "Wavelet-based multifractal analysis of rough surfaces: Application to cloud models and satellite data," Phys. Rev. Lett., vol. 79, pp. 75-79, 1997.

[18] E. Abraham and M. Bowen, "Chaotic stirring by a mesoscale surfaceocean flow," Chaos, vol. 12, no. 2, pp. 373-381, 2002.

[19] V.-K. Gupta and E.-C. Waymire, "A statistical analysis of mesoscale rainfall as a random cascade," J. Appl. Meteorol., vol. 32, pp. 251-267, 1993.

[20] D. Schertzer and S. Lovejoy, "Universal multifractals do exist!: comments on "a statistical analysis of mesoscale rainfall as a random cascade"," J. Appl. Meteorol., vol. 36, pp. 1296-1303, 1997.

[21] J. Grazzini, A. Turiel, and H. Yahia, "Entropy estimation and multiscale processing in meteorological satellite images," in Proc. Int. Conf. Pattern Recognition, vol. 3, 2002, pp. 764-768.

[22] A. Turiel and A. del Pozo, "Reconstructing images from their most singular fractal manifold," IEEE Trans. Image Process., vol. 11, no. 4, pp. 345-350, Apr. 2002.

[23] S. W. Nesbitt, E. J. Zipser, and D. J. Cecil, "A census of precipitation features in the tropics using TRMM: Radar, ice scattering, and lightning observations," J. Clim., vol. 13, pp. 4087-4106, 2000. 